\title{
UNIQUENESS AND NON-UNIQUENESS OF SOLUTIONS OF VLASOV-MCKEAN EQUATIONS
}

\author{
MICHAEL SCHEUTZOW \\ (Received 5 March 1986; revised 29 September 1986) \\ Communicated by T. C. Brown
}

\begin{abstract}
We study the equation $d Y(t) / d t=f(Y(t), E h(Y(t)))$ for random initial conditions, where $E$ denotes the expected value. It turns out that in contrast to the deterministic case local Lipschitz continuity of $f$ and $h$ are not sufficient to ensure uniqueness of the solutions. Finally we also state some sufficient conditions for uniqueness.
\end{abstract}

1980 Mathematics subject classification (Amer. Math. Soc.): 34 F 05, 35 K 22, 60 H 10.

\section{Introduction}

Vlasov-McKean equations of the form

(1) $d X(t)=b(X(t), \mathscr{L}(X(t))) d t+\sigma(X(t), \mathscr{L}(X(t))) d W(t), \quad X(0)=\zeta$ frequently occur as the equation for the dynamics of one component in the mean-field- (or Vlasov-McKean-) limit $[1,4,5,6,8]$. Here $\mathscr{L}$ denotes the law of a random vector, $(W(t))_{t \geqslant 0}$ is an $n$-dimensional Brownian motion, $\zeta$ is an $\mathbf{R}^{d}$-valued random variable and $b$ and $\sigma$ are functions having domain and range such that (1) makes sense. Equation (1) is frequently called "nonlinear stochastic differential equation" since the associated Fokker-Planck (-Vlasov) equation is a nonlinear partial (integro-) differential equation. When proving limit theorems of this type one step (besides tightness and identification of the limit) usually consists of proving pathwise uniqueness of the solution of (1) (see, for example, $[6,8])$. Under certain assumptions (mostly including Lipschitz conditions) particular equations of type (1) or the corresponding Fokker-Planck equations were

(C) 1987 Australian Mathematical Society 0263-6115/87 \$A2.00+0.00 
shown to have a unique solution (in different senses, see $[1,2,4,5,6,7,8]$ ). In many applications however $b$ and/or $\sigma$ are not Lipschitz continuous, but, for example of polynomial type (for example in chemical reaction models, see [6]) and the question arises whether one can still prove pathwise uniqueness. In this paper we provide a partial answer for degenerate equations, where $\sigma \equiv 0$ (like Vlasov equation) and which are of the particular structure

$$
\dot{Y}(t)=f(Y(t), E h(Y(t))), \quad Y(0)=\xi .
$$

We give examples showing that a local Lipschitz condition on either $f$ or $h$ is insufficient for uniqueness of a solution even if the other function satisfies a global Lipschitz condition. These examples are in contrast to the deterministic case (that is, (2) without the expectation in front of $h$ ) where local Lipschitz continuity of $f$ and $h$ are known to be sufficient for uniqueness. The results, although perhaps not too surprising due to the global effect of taking the expected value of $h$, that is, integrating over the whole state space, are still remarkable since the formally approximating system of equations

$$
\dot{Y}_{i}(t)=f\left(Y_{i}(t), \frac{1}{N} \sum_{j=1}^{N} h\left(Y_{j}(t)\right)\right), \quad Y_{i}(0)=\xi_{i}, i=1, \ldots, N,
$$

with $\xi_{i}$ independent and identically distributed, has a unique (local) solution for every finite $N$ if $f$ and $h$ satisfy a local Lipschitz condition. By a (strong) solution of (2) for $0 \leqslant t \leqslant T$ on a probability space $(\Omega, \mathscr{F}, P)$ on which $\xi$ is defined, we mean a stochastic process $(Y(t))_{0 \leqslant t \leqslant T}$ on that space such that $E h(Y(t))<\infty$ for all $0 \leqslant t \leqslant T, \int_{0}^{T} E|h(Y(t))| d t<\infty, Y$ is continuous with probability one and satisfies the integrated equation (2). In the first part we specify a number of conditions on $f$ and $h$ which are insufficient for (2) to have a unique solution by providing counterexamples. In the last part we prove three positive results on uniqueness. More precisely we verify the following table

\begin{tabular}{l|l|l|l}
\hline$h$ & $\begin{array}{l}\text { Lipschitz and } \\
\text { compact support }\end{array}$ & Lipschitz & \multicolumn{2}{c}{ locally Lipschitz } \\
\hline $\begin{array}{l}\text { Lipschitz and } \\
\text { compact support }\end{array}$ & unique (P1, P2, P3) & unique (P1, P2) & unique (P2) \\
\hline Lipschitz & unique (P1, P3) & unique (P1) & CE 1, CE 1' \\
\hline $\begin{array}{l}\text { locally Lipschitz } \\
\text { continuous and } \\
\text { compact support }\end{array}$ & unique (P3) & CE 2 & CE 1, CE 1', CE 2 \\
\hline
\end{tabular}


"CE" stands for "counterexample", "P" stands for "proposition". We omitted the column " $f$ continuous and compact support" since counterexamples are well known in that case. The table holds true for every dimension $d(1 \leqslant d<\infty)$ of the state space of $Y$ and every dimension $n(1 \leqslant n<\infty)$ of the range of $h$.

\section{Counterexamples}

Counterexample 1. To construct the first counterexample let $\left(p_{i}\right)_{i \in \mathbf{N}_{0}}$ be a sequence of strictly positive numbers such that $\sum_{j=1}^{\infty} p_{j}=1$ and $\sum_{j=0}^{\infty} j p_{j}<\infty$. Furthermore let $\left(g_{i}(\cdot)\right)_{i \in N_{0}}$ be a sequence of twice continuously differentiable functions from the interval $\left[0, \frac{1}{2}\right]$ to $\left[0, \frac{1}{2}\right]$ with the properties

(i) $g_{i}(0)=0$.

(ii) $g_{i}^{\prime}(s)>0,0<s<\frac{1}{2}$ (the prime denotes derivative w.r.t. $s$ ).

(iii) There exists some $s_{i}>0$ such that $g_{i}(s)=s^{2}$ for $0 \leqslant s \leqslant s_{i}$.

(iv) $g_{i}\left(\frac{1}{2}\right)=\frac{1}{2}$, and

(v) $\sum_{j=0}^{n} p_{j} g_{j}(t)$ converges to $h(t):=2 t-2 t^{2}$ pointwise (and hence uniformly) on $\left[0, \frac{1}{2}\right]$ as $n \rightarrow \infty$.

To see that such a sequence of functions exists, define

$R_{0}(t):=h(t)=2 t-2 t^{2}, \quad 0 \leqslant t \leqslant \frac{1}{2}$,

$s_{-1}:=\frac{1}{2}$,

$s_{i}:=\min \left(\frac{1}{2}\left(1-\frac{p_{i}}{\sum_{j=i}^{\infty} p_{j}}\right) R_{i}\left(s_{i-1}\right), \frac{1}{2}\left(\frac{R_{i}\left(s_{i-1}\right)}{\sum_{j=i}^{\infty} p_{j}}\right)^{1 / 2}\right) \quad$ and

$R_{i+1}(t):= \begin{cases}R_{i}(t)-p_{i} t^{2}\left(=2 t-\left(2+\sum_{j=0}^{i} p_{j}\right) t^{2}\right), & t \leqslant s_{i}, \\ R_{i}(t)\left(1-\frac{p_{i}}{\sum_{j=i}^{\infty} p_{j}}\right), & t \geqslant s_{i-1},\end{cases}$

and let $R_{i+1}$ be twice continuously differentiable on $\left[0, \frac{1}{2}\right]$ such that $0<R_{i+1}^{\prime}(t)$ $<R_{i}^{\prime}(t)$ for $0<t<\frac{1}{2}$. The inequalities for $R_{i+1}^{\prime}(t)$ are clearly satisfied for $0<t \leqslant s_{i}$ and $s_{i-1} \leqslant t<\frac{1}{2}$ by induction, where $R_{i+1}^{\prime}(t)>0$ for $t \leqslant s_{i}$ follows from $s_{i} \leqslant \frac{1}{4}$ (since $R_{i}(t) \leqslant \frac{1}{2}$ by induction) which implies $R_{i+1}^{\prime}(t)=2-$ $2\left(2+\sum_{j=0}^{i} p_{j}\right) t \geqslant 2-6 t>0$ for $t \leqslant s_{i}$. Note that $s_{i}<s_{i-1}$ since $R_{i}^{\prime}(t) \leqslant 2$ by induction and hence $R_{i}\left(s_{i-1}\right) \leqslant 2 s_{i-1}$.

To show that it is possible to choose $R_{i+1}(t)$ in $\left[s_{i}, s_{i-1}\right]$ such that the inequalities for $R_{i+1}^{\prime}(t)$ hold it is enough to ensure that

$$
0<R_{i+1}\left(s_{i-1}\right)-R_{i+1}\left(s_{i}\right)<R_{i}\left(s_{i-1}\right)-R_{i}\left(s_{i}\right) .
$$


The right inequality is equivalent to

$$
R_{i}\left(s_{i-1}\right)\left(1-\frac{p_{i}}{\sum_{j=i}^{\infty} p_{j}}\right)-R_{i}\left(s_{i}\right)+p_{i} s_{i}^{2}<R_{i}\left(s_{i-1}\right)-R_{i}\left(s_{i}\right)
$$

and hence to $s_{i}^{2}<R_{i}\left(s_{i-1}\right) / \sum_{j=i}^{\infty} p_{j}$, the left one is equivalent to

$$
2 s_{i}-s_{i}^{2}\left(2+\sum_{j=0}^{i} p_{j}\right)<\left(1-\frac{p_{i}}{\sum_{j=i}^{\infty} p_{j}}\right) R_{i}\left(s_{i-1}\right)
$$

which follows from the definition of $s_{i}$. Now $g_{i}(t):=\left(R_{i}(t)-R_{i+1}(t)\right) / p_{i}$ has all the properties (i)-(v). Note that $R_{n}(t)=h(t)-\sum_{j=0}^{n-1} p_{j} g_{j}(t)$ converges to zero at $t=\frac{1}{2}$ and hence, due to $R_{n}^{\prime}(t) \geqslant 0$ and $R_{n}(0)=0$, for all $0 \leqslant t \leqslant \frac{1}{2}$.

Let us now construct a locally Lipschitz continuous function $\phi: \mathbf{R} \rightarrow \mathbf{R}$ such that the equation

$$
\dot{Y}(t)=\phi(Y(t))+E Y(t)-\sum_{j=0}^{\infty} j p_{j}
$$

with $P\{Y(0)=i\}=p_{i}, i \in \mathbf{N}_{0}$, has at least two different solutions on the interval $\left[0, \frac{1}{4}\right]$. Note that the sequence $\left(p_{i}\right)_{i=1,2, \ldots}$ is fixed and therefore $\sum_{j=0}^{\infty} j p_{j}$ is a constant (which could have been incorporated into the function $\phi$ ). Fixing a sequence of functions $\left(g_{i}\right)_{i \in \mathbf{N}_{0}}$ satisfying the assumptions above, define

$$
Y_{i}(t):=i+g_{i}(t), \quad 0 \leqslant t \leqslant \frac{1}{2},
$$

and $\phi: \mathbf{R} \rightarrow \mathbf{R}$ by

$$
\phi(i+t)=g_{i}^{\prime}\left(g_{i}^{-1}(t)\right)-\left(2 g_{i}^{-1}(t)-2\left(g_{i}^{-1}(t)\right)^{2}\right)
$$

for $i \in \mathbf{N}_{0}$ and $0 \leqslant t \leqslant g_{i}\left(\frac{1}{4}\right)$.

Let $\phi(s)=0$ for $s<0$ and define $\phi$ on the intervals $\left[i+g_{i}\left(\frac{1}{4}\right), i+1\right]\left(i \in \mathbf{N}_{0}\right)$ by linear interpolation. The function $\phi$ is well defined since the functions $g_{i}$ are strictly monotonically increasing on $\left[0, \frac{1}{4}\right]$. Obviously $\phi(i)=0$ for all $i \in \mathbf{N}_{0}$ and $\phi$ is continuous. For $0 \leqslant t \leqslant \frac{1}{4}$

$$
\begin{aligned}
\phi\left(Y_{i}(t)\right) & =\phi\left(i+g_{i}(t)\right)=g_{i}^{\prime}(t)-\left(2 t-2 t^{2}\right)=g_{i}^{\prime}(t)-\sum_{j=0}^{\infty} p_{j} g_{j}(t) \\
& =\dot{Y}_{i}(t)-\sum_{j=0}^{\infty} p_{j} Y_{j}(t)+\sum_{j=0}^{\infty} p_{j} j \\
& =\dot{Y}_{i}(t)-\sum_{j=0}^{\infty} p_{j} Y_{j}(t)+\sum_{j=0}^{\infty} p_{j} Y_{j}(0) .
\end{aligned}
$$

Define the process $(Y(t))_{t \in[0,1 / 4]}$ by $P\left\{Y(t)=Y_{i}(t)\right.$ for all $\left.0 \leqslant t \leqslant \frac{1}{4}\right\}=p_{i}$. Then it follows that $Y$ satisfies equation equation (4) with the initial condition $P\{Y(0)=i\}=p_{i}$. 
Since $\phi(i)=0$ for $i=0,1,2, \ldots$ the equation is also solved by the process $\tilde{Y}$ defined by $P\{\tilde{Y}(t)=i$ for all $t \geqslant 0\}=p_{i}$ which clearly has a law different from that of $Y$.

All that remains to show is that the function $\phi$ is not only continuous but in fact locally Lipschitz continuous. It suffices to check the Lipschitz continuity on every interval $\left[i, i+g_{i}\left(\frac{1}{4}\right)\right]$. Differentiating and evaluating at $i+g_{i}(t)$ we get

$$
\phi^{\prime}\left(i+g_{i}(t)\right)=\frac{g_{i}^{\prime \prime}(t)}{g_{i}^{\prime}(t)}-\frac{1}{g_{i}^{\prime}(t)}(2-4 t)
$$

for all $0<t<\frac{1}{4}$. For $0<t \leqslant s_{i}$

$$
\phi^{\prime}\left(i+g_{i}(t)\right)=\frac{2}{2 t}-\frac{1}{2 t}(2-4 t)=2 .
$$

For $s_{i} \leqslant t \leqslant \frac{1}{4}, g_{i}^{\prime}(t)$ is bounded away from zero which shows that $\phi^{\prime}(t)$ is bounded on $] i, i+g_{i}(t)[$ and hence that $\phi$ is locally Lipschitz continuous.

REMARK. The assumption that the $p_{i}$ are (strictly) positive can be relaxed by only requiring that infinitely many of the $p_{i}$ are strictly positive and the others are zero. If however only finitely many are nonzero the construction does not work.

CountereXample $1^{\prime}$. One might suspect that the construction of two different solutions of equation (4) was possible only because $E Y(t)$ is not a (weakly) continuous functional of the law of $Y(t)$. The following generalization of counterexample 1 shows however that this is not true.

Let $h$ be a continuously differentiable function from $\mathbf{R}$ to $\mathbf{R}$ satisfying $h^{\prime}(x)>0$ for all $x \in \mathbf{R}$ ( $h$ can be bounded or unbounded). Furthermore let $\left(p_{i}\right)_{i=0,1,2 \ldots}$ be a sequence of nonnegative numbers which contains infinitely many nonzero elements satisfying $\sum_{j=0}^{\infty} p_{j}=1$ and $\sum_{j=0}^{\infty} p_{j} h(j)<\infty$. Almost the same construction as before shows that

$$
\begin{aligned}
\dot{Y}(t)=\phi(Y(t))+E h(Y(t))-\sum_{j=0}^{\infty} p_{j} h(j), \\
\quad P\{Y(0)=i\}=p_{i}, i=0,1,2, \ldots,
\end{aligned}
$$

has more than one solution on some interval $\left[0, t_{0}\right]$ if $\phi$ is defined in the same way as before, but where the $g_{i}$ satisfy

$$
\sum_{j=0}^{\infty} p_{j}\left(h\left(j+g_{j}(t)\right)-h(j)\right)=2 t-2 t^{2} \quad \text { for } 0 \leqslant t \leqslant t_{0}
$$


instead of $\sum_{j=0}^{\infty} p_{j} g_{j}(t)=2 t-2 t^{2}$ and $g_{i}\left(t_{0}\right) \leqslant \frac{1}{2}$ instead of $g_{i}\left(\frac{1}{2}\right)=\frac{1}{2}$. It is not hard to see that such $g_{i}$ exist and that one can choose any $t_{0}>0$ which is not greater than $\left(1-(1-\min (A, 1))^{1 / 2}\right) / 2$ where $A=\sum_{j=0}^{\infty} p_{j} \min _{j \leqslant s \leqslant j+1} h^{\prime}(s)$ because in this case

$$
\sum_{j=0}^{\infty} p_{j}\left(h\left(j+g_{j}\left(t_{0}\right)\right)-h(j)\right) \geqslant \sum_{j=0}^{\infty} p_{j} \min _{j \leqslant s \leqslant j+1} h^{\prime}(s) \cdot g_{j}\left(t_{0}\right)
$$

which can, by an appropriate choice of the $g_{j}$, assume any value between 0 and $A / 2$, but $A / 2$ is greater or equal to $2 t_{0}-2 t_{0}^{2}$ if $t_{0}$ is chosen in the way above.

COUNTEREXAMPLE 2. We give an example showing that the equation

$$
\dot{Y}(t)=E h(Y(t)), \quad Y(0)=\xi
$$

can have more than one solution if $h$ is locally Lipschitz continuous.

Take a sequence of nonnegative numbers $\left(p_{i}\right)_{i \in \mathbf{N}_{0}}$ which contains infinitely many nonzero members such that $\sum_{j=0}^{\infty} p_{j}=1$. Now let $h$ be any locally Lipschitz continuous function satisfying $\sum_{j=0}^{\infty} p_{j} h(j+u)=(3 u)^{2 / 3}$ where the sum converges uniformly for all $0 \leqslant u \leqslant 1 / 3$. Define $g(t):=t^{3} / 3$ for $0 \leqslant t \leqslant 1$ and $Y_{i}(t)=i+g(t)$. Then $\dot{Y}_{i}(t)=\dot{g}(t)=t^{2}=(3 g(t))^{2 / 3}=\sum_{j=0}^{\infty} p_{j} h(j+g(t))=$ $\sum_{j=0}^{\infty} p_{j} h\left(Y_{j}(t)\right)$, that is, the process $Y$ defined by $P\left\{Y(t)=Y_{i}(t)\right.$ for $\left.0 \leqslant t \leqslant 1\right\}$ $=p_{i}, i \in \mathbf{N}_{0}$ satisfies equation (6) provided $P\{\xi=i\}=p_{i}$ for all $i=0,1,2, \ldots$. On the other hand equation (6) is also solved by $\tilde{Y}(t)=\xi$ for all $t \geqslant 0$ since $\sum_{j=0}^{\infty} p_{j} h(j)=0$.

REMARKs. (i) $h$ can be chosen to be bounded and even to converge to zero at an arbitrary speed (in particular $h$ is then uniformly continuous): Pick a sequence of positive numbers $\left(a_{i}\right)_{i \in \mathrm{N}_{0}}$ converging to zero monotonically. Then we construct a locally Lipschitz continuous nonnegative function $h: \mathbf{R} \rightarrow \mathbf{R}$ such that $\max _{i \leqslant s \leqslant i+1} h(s) \leqslant a_{i}$ for every $i \in \mathbf{N}_{0}$ and such that (6) has more than one solution. Let $t_{0}:=\min \left(\frac{1}{3}\left(\sum_{j=0}^{\infty} p_{j} a_{j}\right)^{3 / 2}, \frac{1}{3}\right)$ and define $h(t)=0$ for $t \leqslant 0$. For $0 \leqslant t \leqslant 1$ let $h$ be Lipschitz continuous, nonnegative and satisfy $\max _{0 \leqslant t \leqslant 1} h(t)$ $\leqslant a_{0}, h(1)=0$ and $0 \leqslant(3 t)^{2 / 3}-p_{0} h(t) \leqslant \sum_{j=1}^{\infty} p_{j} a_{j}$ for all $0 \leqslant t \leqslant t_{0}$. Such a function exists by the definition of $t_{0}$. Continue by defining $h$ in the interval $[1,2]$ such that $h$ is nonnegative, Lipschitz continuous and satisfies $\max _{1 \leqslant t \leqslant 2} h(t) \leqslant a_{1}$, $h(2)=0$ and $0 \leqslant(3 t)^{2 / 3}-\sum_{j=0}^{1} p_{j} h(j+t) \leqslant \sum_{j=2}^{\infty} p_{j} a_{j}$ for all $0 \leqslant t \leqslant t_{0}$ and so forth. It follows that $\sum_{j=0}^{\infty} p_{j} h(j+u)=(3 u)^{2 / 3}$ for $0 \leqslant u \leqslant t_{0}$. The same reasoning as in counterexample 2 shows that there exist at least two solutions on the interval $\left[0, t_{0}\right]$.

(ii) As mentioned in the introduction one main interest in studying McKean equations is due to the fact that they often appear as limiting equations for the motion of one particle in a field of $N$ interacting like particles as $N$ tends to 
infinity. This is also true for the example given above: Consider $N$ particles with positions $\left(Y^{(1, N)}(t), \ldots, Y^{(N, N)}(t)\right)$ having independent $\mathscr{L}(Y(0))$-distributed initial conditions $Y^{(i, N)}(0)$ and satisfying

$$
\dot{Y}^{(i, N)}(t)=\frac{1}{N} \sum_{j=1}^{N} h\left(Y^{(j, N)}(t)\right), \quad i=1, \ldots, N,
$$

with $h$ defined like in the preceding remark. Since $h$ is locally Lipschitz continuous the only solution of $(7)$ is $Y^{(i, N)}(t)=Y^{(i, N)}(0)$ for all $t \geqslant 0$ and $i=$ $1,2, \ldots, N$. So the processes $Y^{(1, N)}(\cdot)$ converge in law to the solution $\tilde{Y}$ of equation (6) but not to the first solution $Y$ !. So the infinite particle system can exhibit a solution that cannot be approximated by the finite dimensional system (7).

(iii) The results of Shiga and Tanaka [8] show (in particular) that the equation $d X(t)=E h(X(t)) d t+d W(t)$ does have a unique solution if $h$ is bounded and measurable. So the noise helps to achieve uniqueness. We conjecture however that one can construct examples of nonuniqueness for the same equation if $h$ is allowed to be unbounded but is locally bounded (or even locally Lipschitz continuous).

COUNTEREXAMPLE 3. The following counterexample is well known. It shows that the equation

$$
\dot{Y}(t)=E h(Y(t)), \quad Y(0)=\xi
$$

can have more than one solution if $h$ is continuous (but not Lipschitz) and has compact support: Let $P\{\xi=0\}=1$ and define

$$
h(x)= \begin{cases}0, & x \leqslant 0, x \geqslant 2 \\ x^{1 / 2}, & 0 \leqslant x \leqslant 1 \\ 2-x, & 1 \leqslant x \leqslant 2\end{cases}
$$

Then (8) is solved by the two deterministic processes $Y_{1}(t)=t^{2} / 4$ and $Y_{2}(t)=0$ for $0 \leqslant t \leqslant 2$.

\section{Some positive results}

Let us now state three positive results about uniqueness of solutions of (2).

Proposition 1. If $f$ and $h$ are Lipschitz-continuous functions and $0<T<\infty$, then there exists at most one (strong) solution of (2) on $[0, T]$. 
Proof. Let $(Y(t))_{0 \leqslant t \leqslant T}$ and $(\tilde{Y}(t))_{0 \leqslant t \leqslant T}$ be two solutions of (2) defined on the same space $(\Omega, \mathscr{F}, P)$ and let $K$ be a Lipschitz constant for both $f$ and $h$. Then

$$
\begin{aligned}
|Y(t)-\tilde{Y}(t)| & \leqslant \int_{0}^{t}|f(Y(s), \operatorname{Eh}(Y(s)))-f(\tilde{Y}(s), \operatorname{Eh}(\tilde{Y}(s)))| d s \\
& \leqslant K \int_{0}^{t}|Y(s)-\tilde{Y}(s)| d s+K \int_{0}^{t}|\operatorname{Eh}(Y(s))-\operatorname{Eh}(\tilde{Y}(s))| d s
\end{aligned}
$$

almost surely. Using the Lemma of Bellman and Gronwall (see Liptser and Shiryayev [3], page 139) we get

$$
\begin{aligned}
|Y(t)-\tilde{Y}(t)| \leqslant & K \int_{0}^{t} e^{K(t-s)} K \int_{0}^{s}|E h(Y(u))-E h(\tilde{Y}(u))| d u d s \\
& +K \int_{0}^{t}|E h(Y(s))-E h(\tilde{Y}(s))| d s .
\end{aligned}
$$

Since the right hand side is deterministic the inequality remains true if we take the expected value on the left side. It follows that there exists a constant $L$ such that

$$
\begin{aligned}
|E h(Y(t))-E h(\tilde{Y}(t))| & \leqslant K E|Y(t)-\tilde{Y}(t)| \\
& \leqslant L \int_{0}^{t}|E h(Y(u))-E h(\tilde{Y}(u))| d u
\end{aligned}
$$

for all $0 \leqslant t \leqslant T$. Applying the Lemma of Bellman and Gronwall once more we get $E h(Y(t))=E h(\tilde{Y}(t))$ for all $0 \leqslant t \leqslant T$ and therefore, from (9) $P\{Y(t)=\tilde{Y}(t)$ for all $0 \leqslant t \leqslant T\}=1$ which proves the assertion.

REMARKs. (i) The proposition and its proof remain true in the multidimensional case, that is, $h: \mathbf{R}^{d} \rightarrow \mathbf{R}^{n}$ and $f: \mathbf{R}^{d} \times \mathbf{R}^{n} \rightarrow \mathbf{R}^{d}$.

(ii) Under the assumptions of Proposition 1 the solution of (2) is also unique in law, that is, the laws of any two solutions $Y$ and $\tilde{Y}$ defined on $(\Omega, \mathscr{F}, P)$ and $(\tilde{\Omega}, \tilde{\mathscr{F}}, \tilde{P})$ respectively with $\mathscr{L}(Y(0))=\mathscr{L}(\tilde{Y}(0))$ coincide. To see this let $m(t):=E h(Y(t))$. Then $\dot{Y}(t)=f(Y(t), m(t)), \quad Y(0)=\xi$ has a solution on $(\Omega, \mathscr{F}, P)$ in the usual sense (see Walter [9], page 82) which is measurable with respect to $\xi$ and which solves (2) in the sense stated in the introduction. Therefore this solution can also be realized (in law) on $(\tilde{\Omega}, \tilde{F}, \tilde{P})$ which, together with proposition 1, proves the assertion. This shows that strong uniqueness always implies uniqueness in law (not only under the assumptions of Proposition 1).

(iii) The assumptions of Proposition 1 do not imply the existence of a solution even if $E|h(Y(0))|<\infty$. Define for example

$$
\begin{array}{ll}
h\left(2^{i}\right)=0, & i=0,1,2, \ldots, \\
h\left(\frac{3}{2} \cdot 2^{i}\right)=2^{i-1}, & i=0,1,2, \ldots, \\
h(t)=0, & t \leqslant 1,
\end{array}
$$


and interpolate $h$ linearly otherwise. Then $h$ is Lipschitz with constant 1. Let $P\left\{Y(0)=2^{i}\right\}=p_{i}, i \in \mathbf{N}_{0}$, such that $\sum_{j=0}^{\infty} 2^{j} p_{j}=\infty$ and define

$$
f(x, y)=x+\alpha(\arctan y+\pi / 2) \quad\left(0<\alpha<(4 \pi)^{-1}\right),
$$

that is, we consider the system

$$
\dot{Y}(t)=Y(t)+\alpha(\arctan E h(Y(t))+\pi / 2) .
$$

Solving this linear equation for $Y$ we get

$$
Y(t)=Y(0) e^{t}+\alpha \int_{0}^{t} e^{-s} \int_{0}^{s} \arctan E h(Y(u))+\pi / 2 d u d s,
$$

but $E h(Y(t)) \geqslant \sum_{j=0}^{\infty} p_{j} h\left(2^{j} e^{t}\right)=\sum_{j=0}^{\infty} p_{j}\left(2^{j} e^{t}-2^{j}\right)=\left(e^{t}-1\right) \sum_{j=0}^{\infty} p_{j} 2^{j}=\infty$ for $0<t \leqslant \ln 5 / 4$ because

$$
0 \leqslant \alpha \int_{0}^{t} e^{-s} \int_{0}^{s} \arctan E h(Y(u))+\pi / 2 d u d s \leqslant \pi / 4 \pi=1 / 4
$$

and $2^{j} e^{t}+1 / 4 \leqslant 2^{j} 5 / 4+1 / 4 \leqslant(3 / 2) 2^{j}$ for $0<t \leqslant \ln 5 / 4$ and $j=0,1,2 \ldots$

(iv) The proof is also valid for (multidimensional) stochastic equations of the form

$$
d Y(t)=f(Y(t), E h(Y(t))) d t+d M(t)
$$

where $M$ is any fixed continuous $L^{2}$-martingale, because the stochastic term drops out in the proof. If in addition to the assumption in Proposition $1 h$ is bounded and $E Y^{2}(0)<\infty$ then uniqueness (and also existence) of the solution of equation (10) follows from a (more general) result of Oelschläger [5]. In fact, if $E|Y(0)|<\infty$, then the usual recursive approximation scheme will give existence of a solution of (10) even without the boundedness condition on $h$.

Proposition 2. If $f$ is locally Lipschitz continuous and $h$ is Lipschitz continuous and has compact support, then for every $0<T<\infty$ there exists at most one strong solution of (2).

Proof. Let $M_{1}$ be chosen such that supp $h \subseteq\left\{x|| x \mid \leqslant M_{1}\right\}$ and let $M_{2}>M_{1}$. Furthermore define $\bar{h}:=\max _{x \in \operatorname{supp} h}|h(x)|$. Since $f$ is bounded on $\{(x, z) \mid$ $\left.|x| \leqslant M_{2},|z| \leqslant \bar{h}\right\}$ there exists some $0<t_{0} \leqslant T$ such that for all measurable functions $(z(t))_{0 \leqslant t \leqslant t_{0}}$ with $\sup _{0 \leqslant t \leqslant t_{0}}|z(t)| \leqslant \bar{h}$ and for all $y_{0}$ satisfying $\left|y_{0}\right| \geqslant$ $M_{2}$, the solution of $\dot{Y}(t)=f(Y(t), z(t)), Y(0)=y_{0}$ satisfies $\inf _{0 \leqslant t \leqslant t_{0}}|Y(t)|>M_{1}$. Now let $\tilde{f}$ be any globally Lipschitz function which agrees with $f$ on the set $\left\{(x, z)|| x\left|\leqslant M_{2},\right| z \mid \leqslant \vec{h}\right\}$. According to Proposition 1

$$
\dot{Y}(t)=\tilde{f}(Y(t), E h(Y(t))), \quad Y(0)=\xi
$$

has at most one solution. 
Let $Y_{1}$ and $Y_{2}$ be two solutions of (2) with $Y_{1}(0)=Y_{2}(0)=\xi$. If we insert $E h\left(Y_{1}(t)\right)$ for $E h(Y(t))$ into (11) we see that the corresponding unique solution $\tilde{Y}(t)$ agrees with $Y_{1}(t)$ on the support of $h$ for $0 \leqslant t \leqslant t_{0}$. In particular $E h(\tilde{Y}(t))$ $=E h\left(Y_{1}(t)\right)$ showing that $\tilde{Y}$ satisfies (11). Since (11) has exactly one solution one can show $E h(\tilde{Y}(t))=E h\left(Y_{2}(t)\right)$ in the same way. So $E h\left(Y_{1}(t)\right)=E h\left(Y_{2}(t)\right)$ for $0 \leqslant t \leqslant t_{0}$ which implies $P\left\{Y_{1}(t)=Y_{2}(t), 0 \leqslant t \leqslant t_{0}\right\}=1$, that is, uniqueness on the interval $\left[0, t_{0}\right]$. Repeating the argument with 0 replaced by $t_{0}$ and $t_{0}$ replaced by $t_{1}=2 t_{0}$ etc. we get uniqueness on $[0, T]$.

REMARK. The proposition and its proof are also valid in the multidimensional case.

Proposition 3. If $f$ is Lipschitz and has compact support w.r.t. the first argument, that is, there exists some $M>0$ such that $f(y, z)=0$ for all $z \in \mathbf{R}$ and $|y| \geqslant M$ and if $h$ is locally Lipschitz continuous then for every $0<T<\infty$ there exists at most one strong solution of (2).

Proof. If $E|h(Y(0))|=\infty$ there is nothing to prove. Otherwise let $\tilde{h}$ be a Lipschitz function which agrees with $h$ inside a ball of radius $M$ and satisfies $E \tilde{h}(Y(0))=E h(Y(0))$. Obviously any solution of (2) satisfies

$$
\dot{Y}(t)=f(Y(t), E \tilde{h}(Y(t))), \quad Y(0)=\xi
$$

since $\dot{Y}(t)=0$ if $|Y(t)| \geqslant M$ and

$$
\begin{aligned}
E \tilde{h}(Y(t))= & E(\tilde{h}(Y(t))|| Y(0) \mid<M) P\{|Y(0)|<M\} \\
& +E(\tilde{h}(Y(t))|| Y(0) \mid \geqslant M) P\{|Y(0)| \geqslant M\} \\
= & E(h(Y(t))|Y(0)|<M) P\{|Y(0)|<M\} \\
& +E(\tilde{h}(Y(0))|| Y(0) \mid \geqslant M) P\{|Y(0)| \geqslant M\} \\
= & E h(Y(t)) .
\end{aligned}
$$

The assertion follows from the fact that equation (12) has at most one solution due to Proposition 1.

REMARK. Again the proposition and its proof are also valid in the multidimensional case.

Final Remark. Instead of (2) some authors treat the (nonlinear) equation

$$
\dot{X}(t)=\int_{\mathbf{R}^{d}} f(X(t), y) d u_{t}(y), \quad u_{t}=\mathscr{L}(X(t))
$$


with $f: \mathbf{R}^{d} \times \mathbf{R}^{d} \rightarrow \mathbf{R}^{d}$ (mostly plus a noise term). If we agree to call $(X(t))_{t \geqslant 0}$ a (strong) solution of (13) if its law $u_{t}=\mathscr{L}(X(t))$ satisfies $\sup _{0 \leqslant t \leqslant T} \int_{\mathbf{R}^{d}}|y| d u_{t}(y)$ $<\infty$ for every $T>0,(X(t))_{t \geqslant 0}$ has continuous paths and (13) holds in its integrated form for almost all paths, then the following table holds true for the function $f$ where uniform (locally) Lipschitz means that the (local) Lipschitz constants can be chosen independently of the other component. Here the uniqueness result is a special case of [2], page 342 .

\begin{tabular}{c|c|c|}
\hline 2nd comp. & \multicolumn{1}{c}{ unif. Lipschitz } & unif. loc. Lipschitz \\
\hline $\begin{array}{c}\text { 1st comp. } \\
\text { unif. Lipschitz } \\
\text { unif. loc. Lipschitz }\end{array}$ & unique & CE 2 \\
\cline { 2 - 3 } & CE 1 & CE 1,2 \\
\hline
\end{tabular}

\section{References}

[1] D. A. Dawson, 'Critical dynamics and fluctuations for a mean-field model of cooperative behavior', J. Statist. Phys. 31 (1983), 29-85

[2] T. Funaki, 'A certain class of diffusion processes associated with nonlinear parabolic equations', Z. Wahrsch. Verw. Gebiete 67 (1984), 331-348.

[3] R. S. Liptser and A. N. Shiryayev, Statistics of random processes I (Springer Verlag, Berlin, 1977)

[4] H. P. McKean, Jr., 'Propagation of chaos for a class of nonlinear parabolic equations', Lecture series in differential equations, Vol. 2, pp. 177-194 (Van Nostrand Reinhold, New York, 1969).

[5] K. Oelschläger, 'A martingale approach to the law of large numbers for weakly interacting stochastic processes', Ann. Probab. 12 (1984), 458-479.

[6] M. Scheutzow, 'Periodic behavior of the stochastic Brusselator in the mean-field limit', Probab. Theory Rel. Fields 72 (1986), 425-462

[7] T. Shiga and H. Tanaka, 'Central limit theorem for a system of Markovian particles with mean field interactions', Z. Wahrsch. Verw. Gebiete 69 (1985), 439-459.

[8] A. S. Sznitman, 'An example of nonlinear diffusion process with normal reflecting boundary conditions and some related limit theorems', preprint, Laboratoire de Probabilités, Paris, February 1983

[9] W. Walter, Gewöhnliche Differentialgleichungen (Springer Verlag, Berlin, 1976).

Fachbereich Mathematik

Universität Kaiserslautern

D-6750 Kaiserslautern

West Germany 